\title{
Condensation of $\mathrm{SiC}$ in Circumstellar Dust Shells of C-Rich Red Giants
}

\author{
ANDREAS GAUGER ${ }^{1}$, JOHN J. KEADY ${ }^{1}$ \\ and ERWIN SEDLMAYR ${ }^{2}$
}

${ }^{1}$ Los Alamos National Laboratory, Theoretical Division
Los Alamos NM, U.S.A.
${ }^{2}$ Institut für Astronomie und Astrophysik, TU Berlin, Germany

Since the late 1960's silicon carbide has been known to be one of the dust components in circumstellar dust shells around carbon-rich red giants due to the attribution of a prominent feature at $11.3 \mu \mathrm{m}$ to solid SiC. From radiative transfer models the fraction of $\mathrm{SiC}$ is inferred to be a few percent by mass, and the shape of the feature can be fitted with the optical properties of $\alpha-\mathrm{SiC}$ powders. Although there exist a few theoretical investigations (e.g. Kozasa \& Sogawa, this conference), the details of the condensation process of $\mathrm{SiC}$ in circumstellar shells are still unknown.

Our investigations show that homogeneous formation of $\mathrm{SiC}$ from the nominal molecule can be ruled out for typical conditions in circumstellar dust shells, due to the low abundance of the $\mathrm{SiC}$ molecule. Condensation of $\mathrm{SiC}$ has to proceed via processes involving more abundant species such as monoatomic silicon, $\mathrm{SiC}_{2}$ and $\mathrm{Si}_{2} \mathrm{C}$. We have considered a condensation scheme in which hydrocarbons $\left(\mathrm{C}_{2} \mathrm{H}, \mathrm{C}_{2} \mathrm{H}_{2}\right)$ and silicon carbide molecules $\left(\mathrm{SiC}_{2}, \mathrm{Si}_{2} \mathrm{C}\right)$ condense onto carbon seeds to form two distinct coexisting solid phases of carbon and $\mathrm{SiC}$. In model calculations for dust-forming outflows this process yields a fraction of solid $\mathrm{SiC}$ of a few percent, in good agreement with the values derived from observations. In the present models nucleation of a separate population of $\mathrm{SiC}$ particles is suppressed because carbon seeds nucleate first, but $\mathrm{SiC}$ nucleation may become important under conditions where carbon condensation is less effective, e.g. in $\mathrm{S}$ stars where the carbon-to-oxygen ratio is close to one.

The calculations were performed on the SGI Cluster at the Institut für Astronomie und Astrophysik. The research at LANL was performed under the auspices of the U.S. Department of Energy. 